\title{
A Novel Mitigation Technique for PAPR in Mimo- OFDMA Using RBWA
}

\author{
Gummadi Suryaprakash $^{1}$, Bala Krishna Konda ${ }^{2}$ \\ ${ }^{1}$ M.Tech (DECS), Department of Electronics \& Communication Engineering, \\ Eluru College of Engineering \& Technology, Eluru, AP, India \\ ${ }^{2}$ Assistant Professor, Department of Electronics \& Communication Engineering, \\ Eluru College of Engineering \& Technology, Eluru, AP, India
}

\begin{abstract}
A new peak-to-average power ratio (PAPR) reduction approach for MIMO-OFDM/A is developed based on the well known Resource block weight algorithm (RBWA). This combines two ideas: 1) time domain signals from "resource blocks" (consisting of several subcarriers) may be linearly combined using precoding weights, transparent to the receiver; 2) the precoding weights can be designed to minimize the modulus variations of the resulting signal, leading generally to a reduction in PAPR. This technique is compatible with various beam forming modes in single antenna and MIMO systems. Simulation results show a noticeable improvement relative to the Selective mapping (SLM) technique with significantly less complexity.
\end{abstract}

Keywords: Beam forming, convex optimization, multiple input multiple output (MIMO), orthogonal frequency division multiplexing (OFDM), Selective mapping technique (SLM).

\section{Introduction}

OFDM is known as one of the most favorable modulation techniques for communication over frequency selective wireless channels, and is widely used in telecommunication standards. A well-known drawback of OFDM is that the amplitude of the time domain signal varies strongly with the transmitted symbols modulated on the subcarriers in the frequency domain, resulting in a ,peaky ${ }^{\text {ee }}$ signal. If the maximum amplitude of the time domain signal is too large, it pushes the transmit amplifier into a non-linear region which distorts the signal resulting in a substantial increase in the error rate at the receiver. Over the past decade, an extensive amount of literature has been dedicated to Peak to Average Power Ratio (PAPR) reduction techniques. These techniques are associated with costs in terms of bandwidth or/and transmit power. Also, most of them require modifications to both the transmitter and the receiver which makes them non-compliant to existing standards. Multiple signal representation methods, such as PTS and selected mapping (SLM) are among the most cited techniques [1], [2]. Extension of these algorithms to multiple antenna (MIMO) systems is not straightforward. Another combined precoding and PAPR reduction technique has been proposed for multiuser MIMO systems with sorted TomlinsonHarashima precoding (sTHP).For more details and further developed techniques on MIMO-OFDM peak reduction see [3] and references therein.

A new technique called CP-PTS is proposed in [4] which is adaptable for different beam forming schemes in standard point to point or multiuser MIMO systems. In this technique, the OFDM subcarriers are grouped into blocks and the phase of each block is changed in a manner similar to the PTS method but without the drawback of sending explicit side information. As long as each block is multiplied with only one phase coefficient, the receiver will perceive this as a channel effect and will compensate for it during the channel equalization process [5]. An extension of CP-PTS to MIMO-
OFDM systems is introduced in[6]. In both cases, a sequential quadratic programming (SQP) algorithm is used to solve the phase optimization problem. The computational complexity of this algorithm can be prohibitive for high data rate and/or low latency communication links. The PAPR weights need to be determined again for every OFDM data block, hence the underlying algorithm should be sufficiently efficient to enable a real-time processing.

In this discussion, the same configuration as CP-PTS is used but instead of solving a non-convex optimization problem, an alternative problem formulation is proposed based on a cost function used in constant modulus algorithms (CMAs). Accordingly, the block-iterative SDCMA algorithm [7] is used to find the precoding PAPR weights. The resulting computational complexity is linear in the number of subcarriers. Furthermore, to make sure that the BER performance of the system is not affected by the PAPR precoding an additional constraint is appended to the CMA objective function which requires the weights to be on the unit circle. Like CP-PTS, the proposed technique is transparent to the receiver; this means that it only affects the base station (BS) and it does not require any signal processing in the mobile station (MS).

The proposed method does not function if the channel estimation exploits the smooth changes of the channel coefficients over the complete OFDM block. However, this assumption is not valid in the modern multiuser systems based on RB assignment [5], [8].

\section{Back Ground Work}

\subsection{Clipping Technique for PAPR Reduction}

In this approach, we can perform time-domain based clipping or frequency-domain based coding. The simplest approach for PAPR reduction is to deliberately clip the amplitude of the signal to a predefined value before 


\section{International Journal of Science and Research (IJSR) \\ ISSN (Online): 2319-7064}

Index Copernicus Value (2013): 6.14 | Impact Factor (2014): 5.611

amplification. However, there are several drawbacks of this approach, such as signal distortion and spectral re-growth. Hence simple clipping is not enough, we have to use coding techniques that are applied to OFDM signals in order to find the optimum threshold for every specific signal. However, this technique works well only when the number of subcarriers is small, because at higher subcarriers, the clipping ratio is to be very low which will lead to more distortion. Clipping is the simplest way to reduce the PAPR, but it causes significant distortion of the signal and increases both the out-of-band radiation and the bit error rate (BER), simulated results shows reduction of PAPR in figs.

\subsection{SLM Technique for PAPR Reduction}

This is described in figure 1. $\mathrm{X}$ is the OFDM data block, $\mathrm{B}$ $(i)^{e c} \mathrm{~s}$ are the phase vectors. $\mathrm{X}(i)^{e c} \mathrm{~s}$ the modified data blocks in the frequency domain. $\mathrm{X}(i)$ are the equivalent time domain blocks.

Where $u=1,2 \ldots \ldots U$. and $N$ is both the number of subcarriers and length of $\mathbf{X}$. Among the modified data blocks $\mathbf{X}(u)$, the one with the lowest PAPR is selected for transmission. It is well known that SLM is more advantageous than PTS if the amount of side information is limited, but the computational complexity of SLM is larger than that of PTS. In order to improve the PAPR reduction performance of SLM scheme, we have to increase the number of phase sequences. The computational complexity of SLM scheme linearly increases as the number of phase sequences increases, which corresponds to the number of IFFTs required to generate the alternative OFDM signals. Even if the SLM scheme is simple and distortion less, sometimes its computational complexity is burdensome.

\section{Transmit Signal Model}

Similar to [6] we consider a generic MIMO-OFDM/A downlink scenario with one base station (BS) employing $M_{t}$ antennas. An OFDM block with $N$ subcarriers is transmitted from each antenna. The $N$ subcarriers include $N_{u}$ useful subcarriers surrounded by two guard bands with zero energy. The useful subcarriers are further grouped into $M$ resource blocks (RBs) each consisting of $N_{b}=N_{u} / M$ subcarriers. Data of one or more users is placed in these RBs and mapped into the space-time domain using an inverse discrete Fourier transform (IDFT) and space-time block coding (STBC). To allow channel estimation at the receivers (mobile stations), each $\mathrm{RB}$ also contains several pilot subcarriers that act as training symbols.

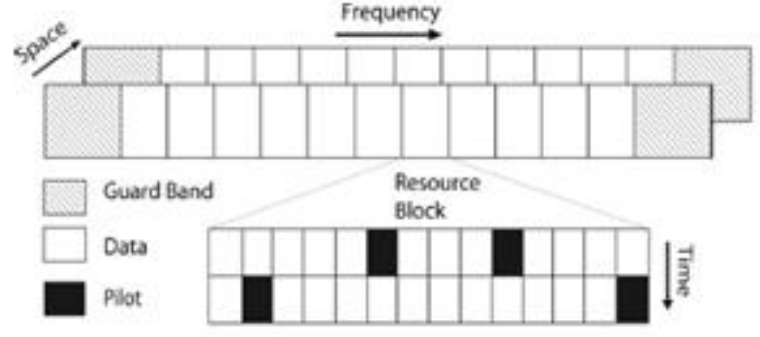

Fig. 1. Data structure of an OFDM block for a MDMO-OFDM/A downlink.

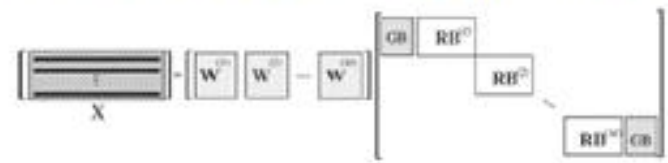

Figure 1: Beam formed MIMO transmit data in frequency domain.

The transmit signal model is illustrated in Fig. 1. It is compatible with the WiMAX standard [8].

Let us first describe the MIMO transmit data model in the frequency domain; for simplicity we consider only a single time block from now on. The data in the q-th RB is a matrix $D^{(q)} \in \square^{M_{t} \times N_{b}}$, it is premultiplied with a corresponding beam forming matrix $W^{(q)} \in \square^{M_{t} \times N_{t}}, q=1, \ldots, M$, resulting in transmit sequences $X^{(q)}=W^{(q) H} D^{(q)}$. Together with guard intervals, they are collected in a matrix $X \in \square^{M_{t} \times N}$, where the $M_{t}$ rows of this matrix represent the $N$ symbols to be transmitted from the $M_{t}$ antennas. The data model is

$$
X=W^{H} D
$$

where $W=\left[W^{(1) H}, \ldots, W^{(M) H}\right]^{H}$, and $D \in \square^{M M_{t} \times N}$ is a block-diagonal matrix with structure as in Fig. 1,which includes guard intervals as well. Matrix $X$ represents the spatial data in the frequency domain.

The time-domain MIMO-OFDM transmit data model is obtained by taking the IDFT of the beam formed data matrix $X$, resulting in

$$
Y=X F^{H}=W^{H} D F^{H}
$$

where $F^{H} \in \square^{N \times N}$ denotes the IDFT matrix, and $Y \in \square^{M_{t} \times N} \quad$ contains the resulting transmit OFDM sequences for each of the $M_{t}$ antennas. Let us further denote the time-domain data matrix $B=D F^{H}$; this is a full matrix. Accordingly, the beam formed OFDM block can be expressed as

$$
Y=W^{H} B
$$

Denote the total power (or energy) in the data matrix $D$ by $P_{d}:=\|D\|_{F}^{2}=\|\operatorname{vec}(D)\|^{2}=: \alpha N_{t}, \quad$ where $\quad N_{t}=N M_{t}$. Function vec $(D)$ creates a column vector whose elements are the columns of the matrix $D . N_{t}$ is the total number of 


\section{International Journal of Science and Research (IJSR) \\ ISSN (Online): 2319-7064}

Index Copernicus Value (2013): 6.14 | Impact Factor (2014): 5.611

subcarriers or samples to be sent from all $M_{t}$ antennas, and $\alpha$ is defined as the average transmit power per sample (including the zero power guard bands). If we assume that the beam forming matrix $W$ consists of orthonormal matrices $W^{(q)}$, then applying beam forming and the IDFT does not change the total transmit power.

\section{Proposed Precoding Scheme}

The IDFT operation in (2) leads to a large dynamic range of the resulting time-domain OFDM signal. PAPR is a common metric to measure the distortion caused by probable high peak of the OFDM signal and for a MIMO-OFDM block $Y$ we define

$$
\operatorname{PAPR}(Y)=\frac{\alpha N_{t}\|\operatorname{vec}(Y)\|_{\infty}^{2}}{\|\operatorname{vec}(Y)\|_{2}^{2}}
$$

Clearly, the lowest PAPR is achieved for a constant modulus (CM) signal, for which the infinity norm is equal to the average power of the sequence.

The main idea in [4], [6] is to design a precoding matrix to transform the OFDM symbols in $Y$ to a favorable signal $S$ with lower PAPR (ideally a CM signal). This precoding matrix $\Omega$ needs to fulfill the following requirements:

1) Reduce the dynamic range of the OFDM block,

2) Preserve the beam forming property,

3) Be transparent to the receiver,

4) Not impact the bit error rate (BER).

To satisfy the second and third constraint, we are allowed to pre multiply each $\mathrm{RB}, D^{(q)}$, with a diagonal scaling matrix $\Omega^{(q)}$. To the receiver, this will appear as a fading channel effect. To not affect the BER, the scaling should be uni modular (phase only). Equivalently, a diagonal (uni modular) precoding matrix $\Omega \in \square^{M M_{t} \times M M_{t}}$ is applied to $D$. The resulting MIMO-OFDM transmit matrix (replacing $Y$ ) is

$$
S=W^{H} \Omega D F^{H}
$$

If we define $\omega=\operatorname{vec} \operatorname{diag}(\Omega)$, then the PAPR reduction problem is to design $\omega$ as

$$
\min _{\omega}\|\operatorname{vec}(S)\|_{\infty}^{2} \quad \text { s.t. } \quad\|\operatorname{vec}(S)\|_{2}^{2}=P
$$

where $P=\alpha N_{t}$ is a fixed total transmit power. This problem is not convex because nonlinear equality constraints can rarely be expressed in a convex form. The approach in [4], [6] was to solve a series of quadratic convex sub problems iteratively. Although this does not solve the original problem in (6) exactly, the results were excellent compared to other techniques, and attractive as the method is transparent to the receiver and does not distort the transmit signals. Unfortunately, this approach is yet too complex for real time applications.

\section{Proposed RBA Approach}

\subsection{Formulation as a Resource Block Problem}

Using properties of Kronecker products, we can rewrite $S$ in (5) as

$$
s=\operatorname{vec}(S)=(\bar{B} \circ W)^{H} \operatorname{vecdiag}(\Omega)=: A \omega
$$

where $A \in \square^{N_{t} \times M M_{t}}, D F^{H}=B \in \square^{M M_{t} \times N}, \bar{B}$ denotes the complex conjugate of $B$, and denotes the Khatri-Rao product (column-wise Kronecker product). The vecdiag $(D)$ creates a column vector whose elements are the main diagonal of the matrix $D$. The optimization problem (6) becomes

$$
\min _{\omega}\|A \omega\|_{\infty}^{2} \quad \text { s.t. } \quad\|A \omega\|_{2}^{2}=\alpha N_{t}
$$

We now propose an alternative formulation of this problem, by replacing the infinity norm by the average deviation of the OFDM block from a constant modulus signal. Ideally, the resulting $S$ will be close to a CM signal, and hence have close-to optimal PAPR. The corresponding cost function is

$$
J(\omega)=\left\|A \omega \square \overline{(A \omega)}-\alpha 1_{N_{t}}\right\|_{2}^{2}=\sum_{n=1}^{N_{t}}\left(\omega^{H} a_{n} a_{n}^{H} \omega-\alpha\right)^{2}
$$

Here, the vector $a_{n}^{H}, n=1, \ldots, N_{t}$ represents the n-throw of matrix $A$, the column vector $1_{N_{t}}$ is a vector with all entries equal to 1 and dimension $N_{t}$, and $\square$ denotes the Schur-Hadamar product (point wise multiplication).

This formulation is similar to the well-known "CMA(2,2)" cost function for adaptive blind equalization or blind beam forming, and can be solved efficiently using available iterative algorithms. The matrix $A$ plays the role of the data matrix in the usual CMA context, whereas $\omega$ plays the role of the beam forming vector. The original CMA cost function is expressed in terms of an expectation operator; the present "deterministic" formulation is similar to the Steepest Descent CMA (SDCMA) in [7].

\subsection{Steepest-Descent RBA (SDRBA)}

The SDRBA is a block-iterative algorithm in which we act on the full data matrix $A$ and $\omega$ update until it converges. The derivation of the block SDRBA is straightforward when the statistical expectation in original formula in [7] is replaced by an average over a block. For the i-th iteration, we start from the current estimate $\omega^{i}$ and compute:

$$
\begin{aligned}
& \hat{s}=A \omega^{i} \\
& \hat{e}^{i}=\left(\begin{array}{cc}
\hat{s}^{i} \square \hat{S}^{i} \\
\hat{S}^{i}
\end{array}\right)-\alpha 1 N_{t} \\
& \hat{S}_{e}=\hat{s}^{i} \square e^{i} \\
& \omega^{i+1}=\omega^{i}-\mu \nabla J\left(\omega^{i}\right)=\omega^{i}-\mu A^{T} \hat{S}_{e}
\end{aligned}
$$




\section{International Journal of Science and Research (IJSR) \\ ISSN (Online): 2319-7064}

Index Copernicus Value (2013): 6.14 | Impact Factor (2014): 5.611

Here, is a suitable step size, and $\hat{S}_{e}$ is the update error. The maximal step size $\mu$ could be defined as a scale independent parameter in relation to the signal power in $A$. To keep the solution unchanged as $A$ scales, $\mu$ needs to be divided by factor $\alpha^{2}, \mu=\mu^{\prime} / \alpha^{2}$. For convergence, the algorithm is initialized with $\omega^{0}=1$ (although other choices are possible). The algorithm should be run until the cost function $J(\omega)$ converges; in practice convergence is fast and the algorithm is run for a fixed small number of iterations.

To satisfy the power constraint in (6), we can simply scale the resulting $\omega$ after convergence. If $s=A \omega$ is indeed a constant modulus signal, then $\|s\|_{2}^{2}=\alpha N_{t}$, and the power constraint is inherently already satisfied. Thus, the scaling is expected to be close to 1 and could be omitted in practice (it has no effect on the cost function $\operatorname{PAPR}(S)$ ).

A difference with the standard RBA is that, here, a good solution does not necessarily exist. The usual application of CMA is for a linear combination of constant modulus sources for which, without noise, a perfect beam former exists. The present situation could be said to correspond to a very noisy source separation situation. Note that, also for other methods, there are no existence results for PAPR reduction.

\subsection{Unit-Circle RBWA (UC-RBA)}

In SDRBA, the computed $\omega$ has no constraints and may have some small entries. These are equivalent to a (broad) null in the channel which will affect the BER performance. Ideally, we should restrict the entries of $\omega$ to take only unimodular values: $\omega_{m}=e^{j \phi_{m}}, m=1, \ldots, M$, and add this constraint to the optimization problem (8). In order to restrict the solution to be on the unit circle, a normalization step is added to each iteration after(12):

$$
\omega^{i+1}=\omega^{i+1} \varnothing\left|\omega^{i+1}\right|
$$

where $\varnothing$ denotes point wise division, and $|\cdot|$ takes the absolute value of each entry of the vector argument. This alternative updating algorithm is called Unit Circle RBA (UC-RBA) since (13) projects the solution of RBA to a unit circle at each iteration.

\subsection{Computational Complexity}

The complexity of the SDRBA algorithm in (12) is dominated by the matrix products $A^{T} \hat{S}_{e}$ and $A \omega^{i}$. The resulting complexity is approximately $2 N M M_{t}^{2}$ per iteration (linear in the number of subcarriers). UC-RBA has the same complexity.

In conventional PTS [2], each RB (sub-block in PTS context) is weighted with a phase shift in such a way that the summation of sub-blocks produces an OFDM sequence with a smaller PAPR [2]. The phase weights are selected by an exhaustive search among a discrete set of phases, and are sent as side information to the receiver. Accordingly, all combinations of the $M$ available phase weights need to be calculated and then multiplied with an IDFT summation matrix, which has the same size as matrix $B$. Finally, one sequence with the least PAPR metric is chosen with the corresponding phase weights. The complexity of the exhaustive search is calculated for the simplest set of only two phases $\left\{ \pm 1=e^{ \pm^{j \pi} / 2}\right\}$ and $M \quad$ RBs as $2^{M} N M$ multiplications and $2^{M}$ comparisons. For $\mathrm{CP}$ PTS, the complexity is $O\left(M^{3}\right)$; the exact expression for complexity is derived in [4].

\section{Simulation Results}

In WiMAX, one RB spans $N_{b}=14$ sub-carriers over two OFDM symbols in time, containing 4 pilots and 24 data symbols. For a $10 \mathrm{MHz}$ system, there are a total of $M=60$ RBs [8]. In agreement with this WiMAX setting, the proposed PAPR reduction technique is simulated for an OFDM block of size $N=1024$ including $M N_{b}=840$ data subcarriers with QPSK modulation and 92 guard subcarriers at each end of the band. The number of MIMO transmit antennas is either $M_{t}=1,2$ or 4 , as will be indicated. The various techniques are evaluated using the complementary cumulative density function (CCDF), which denotes the probability that the PAPR of a data block exceeds the argument of the function
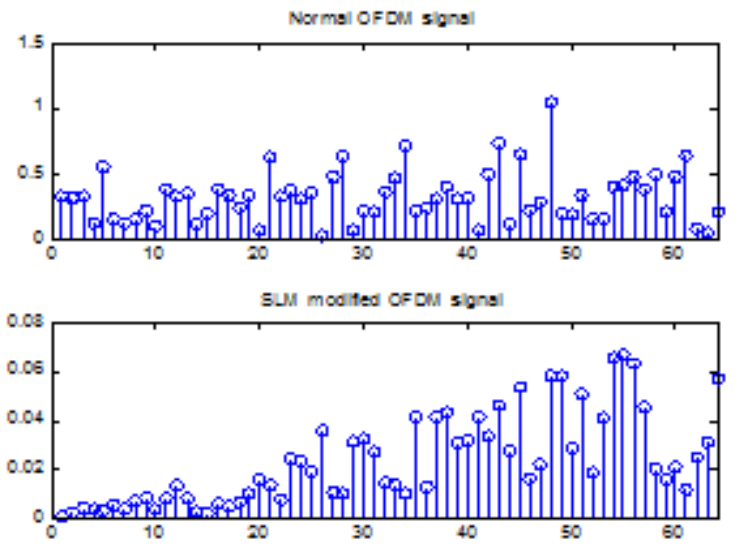

Figure 2: PAPR in OFDM with SLM

From the figure PAPR for normal OFDM is $20.566 \mathrm{~dB}$ and where as PAPR with SLM is $16.0764 \mathrm{~dB}$. 


\section{International Journal of Science and Research (IJSR) \\ ISSN (Online): 2319-7064}

Index Copernicus Value (2013): 6.14 | Impact Factor (2014): 5.611

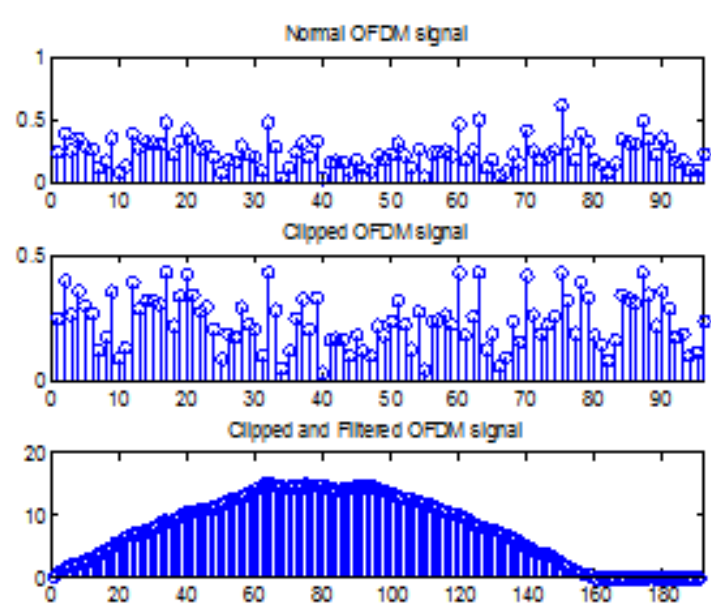

Figure 3: Clipping technique for PAPR

From the figure PAPR for normal OFDM is $5.2755 \mathrm{~dB}$ and where as PAPR with Clipping technique is $2.5949 \mathrm{~dB}$.

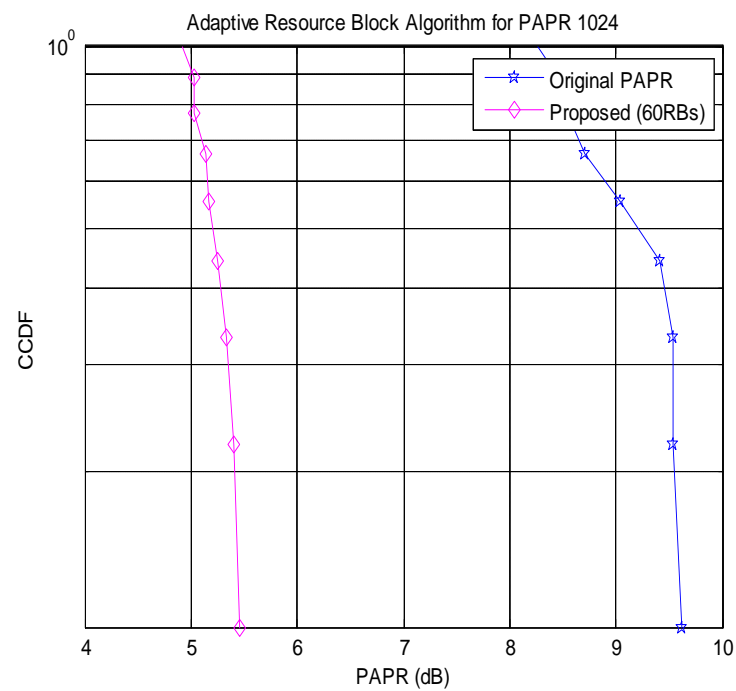

Figure 5: Proposed PAPR technique in OFDMA

From the fig5 Original PAPR OFDM signal is $20.2752 \mathrm{db}$ and PAPR clipped OFDM is $10.053 \mathrm{db}$, where as for SLM, the PAPR calculated is 13.9481 But using Adaptive Resource Block algorithm the PAPR calculated is 5.0300. and the effiency is $94.97 \%$ compared to SLM i.e. $86.05 \%$

\section{Conclusions}

This project proposes a optimization technique to dynamically Reduce the PAPR of OFDM signal using Adaptive Resource Block Algorithm compared to Clipping and SLM methods. It has been observed that this method has better PAPR and computational complexity reduction performances without the degradation of the BER. These results will help to design an efficient PAPR reduction in OFDM and will increase the efficiency of utilization of spectrum and provide large economic and social benefits.

\section{References}

[1] S. H. Han and J. H. Lee, "An overview of peak-toaverage power ratio reduction techniques for multicarrier transmission," IEEE Wireless Commun. Mag., vol. 12, pp. 56-65, Apr. 2005.

[2] S. Muller and J. Huber, "OFDM with reduced peak-toaverage power ratio by optimumcombination of partial transmit sequences," Electron. Lett., vol. 33, pp. 368369, Feb. 1997.

[3] C. Siegl and R. F. Fischer, "Selected basis for PAR reduction in multi-user downlink scenarios using latticereduction-aided precoding," EURASIP J. Adv. Signal Process., Jul. 2011.

[4] S. Khademi, T. Svantesson, M. Viberg, and T. Eriksson, "Peak-to-average- power-ratio (PAPR) reduction in WiMAX and OFDM/A systems," EURASIP J. Adv. Signal Process., Aug. 2011.

[5] Y. Shen and E. Martinez, "WiMAX channel estimation: Algorithms and implementations," Application Note, Freescale, 2007.

[6] K.Murali and N Ramesh Babu "Performance Analysis of CP-OFDM with Different fading channels with Energy Efficient Binary Power Control” ISTP-JREEE in (Volume 3, Issue 4 July 14)

[7] S. Khademi, A.-J. van der Veen, and T. Svantesson, "Precoding technique for peak-to-average-power-ratio (PAPR) reduction in MIMO OFDM/A systems," in , 2012 IEEE Int. Conf. Acoustics, Speech and Signal Processing (ICASSP), Mar. 2012, pp. 3005-3008.

[8] K.Murali and P.Dhana Lakshmi "A Novel approach for reduction of foliage loss in wireless communications through molecular nano communications"in IJAST, ISSUE 3, Vol. 6, Nov13 -ISSN 2249-9954). (IF 2.94)

[9] J. Treichler and B. G. Agee, "A new approach to multipath correction of constant modulus signals," IEEE Trans. Acoust., Speech, Signal Process., vol. 31, pp. 459-471, Apr. 1983.

[10] K.Murali ,M.Sucharitha etc.al“" A Novel design of Time Varying Analysis of Channel estimation Methods of OFDM" in International Journal of Management, IT \& Engineering (ISSN: 2249-0558) June 2012 Volume 2, Issue 6 (ICV 9.00)

[11] J. Wang, Z. Lan, R. Funada, and H. Harada, "On scheduling and power allocation over multiuser MIMOOFDMA: Fundamental design and performance evaluation WiMAX systems," in IEEE Int. Symp. Personal, Indoor and Mobile Radio Communications, 2009, pp. 2752-2756. 